\title{
Encapsulated Checkpoint Blocker Before Chemotherapy: The Optimal Sequence of Anti-CTLA-4 and Doxil Combination Therapy
}

This article was published in the following Dove Press journal: International Journal of Nanomedicine

\author{
Reza Alimohammadi' \\ Razieh Alibeigi ${ }^{2}$ \\ Amin Reza Nikpoor ${ }^{3}$ \\ Ghanbar Mahmoodi Chalbatani ${ }^{4}$ \\ Thomas J Webster (D) ${ }^{5}$ \\ Mahmoud Reza Jaafari (iD) $6,7, *$ \\ Seyed Amir Jalali ${ }^{1} *$ \\ 'Department of Immunology, School of \\ Medicine, Shahid Beheshti University of \\ Medical Sciences, Tehran, Iran; ' ${ }^{2}$ epartment \\ of Immunology, School of Medicine, \\ Mashhad University of Medical Sciences, \\ Tehran, Iran; ${ }^{3}$ Molecular Medicine Research \\ Center, Hormozgan Health Institute, \\ Hormozgan University of Medical Sciences, \\ Bandar Abbas, Iran; ${ }^{4}$ Department of \\ Immunology, School of Medicine, Tehran \\ University of Medical Sciences, Tehran, Iran; \\ ${ }^{5}$ Department of Chemical Engineering, \\ Northeastern University, Boston, MA \\ 021 I5, USA; ${ }^{\circ}$ Nanotechnology Research \\ Center, Pharmaceutical Technology Institute, \\ Mashhad University of Medical Sciences, \\ Mashhad, Iran; ${ }^{7}$ Department of \\ Pharmaceutical Nanotechnology, School of \\ Pharmacy, Mashhad University of Medical \\ Sciences, Mashhad, Iran \\ *These authors contributed equally to this \\ work
}

Correspondence: Seyed Amir Jalali Department of Immunology, Medical School, Shahid Beheshti University of Medical Sciences, Tehran 19857I-7443, Iran Tel +98 2I-238 72545

$\mathrm{Fax}+9821-22439970$

Email jalalia@sbmu.ac.ir

Mahmoud Reza Jaafari

Biotechnology Research Center,

Nanotechnology Research Center, School

of Pharmacy, Mashhad University of Medical

Sciences, Mashhad 91775-1365, Iran

Tel +98 5I-38823255

Fax+98 5I-3882325I

Email Jafarimr@mums.ac.ir
Introduction: Today, a new paradigm has emerged for cancer treatment introducing combination therapies. Doxil, a liposomal doxorubicin serving as a chemotherapeutic agent, is an effective immunogenic killer of cancer cells. Anti-CTLA-4 has been approved for the treatment of some cancers, including melanoma, but side effects have limited its therapeutic potential.

Methods: In this study, two approaches were utilized to increase treatment efficiency and decrease the side effects of anti-CTLA-4, combining it with chemotherapy and encapsulation in a PEGylated liposome. A different sequence of anti-CTLA-4 and Doxil was assessed in combination therapy using non-liposomal and liposomal anti-CTLA-4.

Results: Our results showed that liposomal anti-CTLA-4 reduced the size of established tumors and increased survival in comparison with non-liposomal anti-CTLA-4 in a wellestablished B16 mouse melanoma model. In combination therapy with Doxil, only the administration of anti-CTLA-4 before Doxil showed synergism in both non-liposomal and liposomal form and increased the $\mathrm{CD}^{+}$/regulatory $\mathrm{T}$ cell ratio.

Discussion: In summary, our results demonstrate the potential of utilizing a nanocarrier system for the delivery of checkpoint blockers, such as anti-CTLA-4 which further showed potential in a combination therapy, especially when administered before chemotherapy.

Keywords: chemotherapy, anti-CTLA-4, checkpoint blockers, immunotherapy, liposome

\section{Introduction}

Cancer cell death induced by chemotherapy is considered as a silent immunogenic manner. However, specific chemotherapeutics (such as doxorubicin, oxaliplatin, UVirradiation, anthracyclines, etc.) show a strong ability to induce Immunogenic Cell Death (ICD) of cancer cells. ICD generates a series of signals (e.g., release or exposure of High Mobility Group Box 1 (HMGB1), ATP, CXCL10 and Calreticulin (CALR)) which further stimulate the immune system to activate it against tumor cells. ${ }^{1-3}$ The cell death caused by chemotherapy can increase the access of tumor antigens to antigen-presenting cells. In addition, chemotherapy can further enhance the immune response against cancer by reducing suppressive activity from regulatory cells. ${ }^{4}$

Along this line, doxorubicin is a common chemotherapy agent used for a wide range of cancers (e.g., sarcoma, lung, breast, etc.). Previously, it has been considered as a cytotoxic agent with direct cell-killing effects by intercalating DNA and topoisomerase inhibition. Recently, doxorubicin has been shown to induce effective ICD and leading to a boost in immune system response and T-cell infiltration into the tumor. ${ }^{5}$ Encapsulation of doxorubicin in PEGylated liposomes (termed Doxil) 
drastically changes the pharmacokinetic properties of doxorubicin, increasing tumor accumulation, and decreasing accumulation in healthy tissues; thus, side effects diminish when using Doxil over doxorubicin. ${ }^{6}$

Besides the beneficial effect of ICD inducers on immune cell activation, expression of checkpoint receptors (such as CTLA-4) increases following chemotherapy. ${ }^{7}$ Combination therapy with checkpoint blockers is a promising strategy to overcome the immune system inhibitory effect of chemotherapy. Among chemotherapy agents, those with the ability to induce ICD are the best to combine with blockers of checkpoint receptors, as Pfirschke et al reported that immunogenic chemotherapy sensitizes the tumor to checkpoint blockers. ${ }^{8}$ Immune checkpoint receptors, mainly CTLA-4 and programmed death-1 (PD-1) and their corresponding ligands, transmit a negative signal to T-cells and have an essential role in tumor cell evasion from the immune system. ${ }^{9}$

CTLA-4 has a crucial inhibitory role in early T-cell activation and proliferation. The first blocking antibody was approved for advanced metastatic melanoma (MM) by the US Food and Drug Administration (FDA) and the European Medical Agency (EMA) in 2011, which came to the clinic almost a decade ago after a series of successful trials. ${ }^{10,11}$ Besides its great response in the clinic, immunerelated adverse events (irAEs) mainly in the pituitary gland, liver, kidneys, and skin limited its therapeutic dosage and widespread clinical application. ${ }^{12-14}$ One approach for reducing side effects and increasing tumor-specific accumulation is by encapsulating anti-CTLA-4 in a nanocarrier. In our previous research, we showed that the encapsulation of anti-CTLA-4 in PEGylated liposomes increased its accumulation in tumors and provided a greater anti-tumor response in comparison with free anti-CTLA-4. ${ }^{15,16}$

In the present study, we evaluated its anti-tumor response by testing non-liposomal and liposome-encapsulated antiCTLA-4 in a B16 large established mouse tumor melanoma model, either in the form of monotherapy or in combination with Doxil. We also assessed the effect of the sequence of administration of anti-CTLA-4 and Doxil as a combination therapy.

\section{Materials and Methods}

\section{Materials}

Hydrogenated soya phosphatidylcholine (HSPC) and Methoxypolyetheleneglycol (Mw 2000) distearylphosphatidylethanolamine (mPEG2000-DSPE) were purchased from Lipoid (Ludwigshafen, Germany). Cholesterol was purchased from Sigma-Aldrich (St. Louis, MO). Commercially available Caelyx ${ }^{\circledR}$ was purchased from the Behestan Darou Company (Tehran, Iran). The IgG antimouse CTLA-4 blockade monoclonal antibody (Clone: 9H10) was purchased from Bioxcell (NH, USA). Pe-Cy5labeled Rat anti-mouse CD4 antibody, PE-labeled Rat antimouse CD8a antibody, PE-labeled Rat anti-mouse CD25 antibody, Alexa-flour labeled Rat anti-mouse Foxp3 antibody, APC labeled anti-mouse CD 45 antibody, NIR Zombie die (for live/Dead discrimination), as well as appropriate isotype control antibodies and a TrueNuclear ${ }^{\mathrm{TM}}$ transcription factor buffer set were purchased from Biolegend (CA, San Diego). Collagenase type I, RPMI 1560 medium, and Fetal Bovine Serum (FBS) was purchased from Gibco (NY, USA). Dnase type I was purchased from Roche (USA). The remaining chemical solvents and reagents were chemical grade.

\section{Mice}

Six to eight weeks old female C57BL/6 mice were purchased from the Pasteur Institute of Iran (Tehran, Iran). The mice were housed at the standard animal house under controlled conditions of $23^{\circ} \mathrm{C}$ (room temperature) and a relative humidity of $65 \%$ in $12 / 12$ hour light/dark cycles with free access to water and rodent chow. All animal experiments were handled in accordance with the Helsinki declaration following approval by the Institutional Ethical Committee and Research Advisory Committee of Shahid Beheshti University of Medical Sciences (dated June. 20, 2016; proposal code 930,574). Welfare of the laboratory animals was based on guideline of the Institutional Animal Care and Use Committee at Shahid Beheshti University of Medical Sciences.

\section{Cell Lines and Tumor Inoculation}

A B16/F10 melanoma cell line was purchased from the Pasteur Institute of Iran (Tehran, Iran) and maintained in Dulbecco's Modified Eagle's media (DMEM) supplemented with $10 \%(\mathrm{v} / \mathrm{v})$ heat-inactivated fetal calf serum (FCS) and $100 \mathrm{IU} / \mathrm{mL}$ penicillin. Cell viability was assessed by a trypan blue dye exclusion before the experiment. For tumor inoculation, $5 \times 10^{5}$ cells were injected subcutaneously in the right flank of each mouse.

\section{Liposome Preparation}

Liposomes were prepared by thin lipid film hydration plus extrusion. Briefly, a stock chloroform solution of 
ingredients for PEGylated liposomes: HSPC, mPEG2000DSPE, and cholesterol (48 $\mathrm{mM}$ total lipid concentration, the molar ratio of $55 / 5 / 40$ ), dried by a rotary drier. The lipid film was then hydrated with $10 \mathrm{mM}$ phosphatebuffered saline (PBS) at a $\mathrm{pH} 7.4$ containing $1 \mathrm{mg} / \mathrm{mL}$ of the anti-CTLA-4 monoclonal antibody. The resulting liposomes were incubated overnight at $4^{\circ} \mathrm{C}$ then liposomes were purified by two different purification methods: chromatography and dialysis. Results showed no significant differences between the two methods. Dialysis was used to purify the antibody-entrapped liposomes from free ones for the rest of the studies. A detailed protocol for synthesis of PEGylated liposomes containing anti-CTLA-4 has been described previously. ${ }^{16}$

\section{Liposome Characterization}

The particle size, polydispersity index (PDI), and zeta potential of the liposomes were measured by a Zetasizer instrument (Nano-ZS; Malvern, UK). Particle sizes were reported as $Z$ averages mean \pm standard deviation, and PDI and values are presented from triplicate measurements for each formulation. Zeta potentials were reported as the mean \pm zeta deviation.

The encapsulation efficiency of the anti-CTLA-4 antibody into liposomes was measured using 20 to $2000 \mu \mathrm{g} / \mathrm{mL}$ of protein detection BCA commercial kit (Thermo Scientific, Rockford, IL, USA). In order to measure the concentrations of entrapped anti-CTLA-4 antibodies into liposomes, the prepared liposomes were dialyzed to purify the antibody-entrapped liposomes from free antibodies. Finally, the encapsulation efficiency percent was calculated by the following formula: $=$ (antibody concentration after purification of liposomes/antibody concentration before purification of liposomes) $\times 100$.

\section{Treatment Experiment}

Ten days after tumor challenges, mice with large established tumors were randomized into eight different treatment receiving groups as follows: 1) non-liposomal anti-CTLA-4 antibody, 2) anti-CTLA-4 PEG-liposomes, 3) Doxil, 4) concomitant non-liposomal anti-CTLA-4 blockade and Doxil, 5) anti-CTLA-PEG-liposomes before Doxil, 6) non-liposomal anti-CTLA-4 Antibody after Doxil, 7) non-liposomal antiCTLA-4 blockade antibody before Doxil and 8) PBS. Mice were injected with a single dose of $5 \mathrm{mg} / \mathrm{kg}$ Doxil on day 10 (group 3)/day11 (groups 4and 6) or day 20 (groups 5and 7) after tumor inoculation (all injections were intra venous via mice lateral tail veins). Mice receiving the anti-CTLA-4
Antibody were injected with three-doses of antibody (100 $\mu \mathrm{g}$ ) on days 11, 15 and 19 after tumor inoculation (in groups 1, 5, 6 and 7). A PEG-liposomal anti-CTLA-4 antibody that contained $100 \mu \mathrm{g}$ of the anti-CTLA-4 antibody was injected on day 11, 15 and 19 after tumor inoculation (in groups 2 and 4). Control mice received $50 \mathrm{~mL}$ of PBS alone for each scheduled injection day (Figure 1A).

\section{Tumor Size and Survival}

Tumor size and body weight (Figure S1) were measured two times a week during the experiment, and tumor volume was calculated as follows: $(\mathrm{a} \times \mathrm{b} \times \mathrm{c})$ (length, width and height) $\times 0.5 \mathrm{~mm}^{3}$. The time to reach the endpoint (TTE) criteria of mice were as follows: finding them dead, or tumor growth more than $2000 \mathrm{~mm}^{3}$, or a decrease in body weight more than $15 \%$ of the initial weight or declining health or visible sign of sickness. TTE and tumor growth delay (\%TGD) for each mouse was calculated by our previous study (15).

\section{Flow Cytometry Analysis of Drainage Lymph Node, Tumor-Infiltrating Lymphocytes, and Spleen}

The flow cytometry analysis was done on tumor-infiltrated lymphocytes (TIL), drainage lymph node, and spleen by a BD FACSlyric flow cytometer (Becton Dickinson, USA). Twenty-two days post tumor challenge, three days after the last scheduled injection, three mice from each group were sacrificed. The tumor tissues of mice were removed and washed with $0.1 \mathrm{mM}$ PBS $(\mathrm{pH} 7.4)$ and then minced into small pieces. For tumor digestion, we diced tumor tissues followed by incubation in RPMI 1640 containing collagenase type I $(2 \mathrm{mg} \backslash \mathrm{mL})$ and DNAse type I $(20 \mathrm{IU} / \mathrm{mL})$ medium for 1 hour at $37^{\circ} \mathrm{C}$ with gentle mixing. Then, the cells were filtered through a cell strainer with a pore size of $70 \mu \mathrm{m}$ (BD Falcon, USA) and centrifuged at $1500 \mathrm{rpm}$ for $10 \mathrm{~min}$. The resulting pellet was resuspended in flow cytometry staining buffer (PBS containing 5\% fetal bovine serum) for further flow cytometry analysis. The drainage lymph nodes and spleen were removed and minced into small pieces and directly passed through a nylon mesh cell filter with a pore size of $70 \mu \mathrm{m}$, followed by centrifugation at $1500 \mathrm{rpm}$ for 10 min. The TILs, DLNs cells, and spleen cells were analyzed by flow cytometry for the expression of CD4, CD8, CD25, and Foxp3 in the live population. After staining with Zombie NIR for live/dead discrimination, PE-Cy5 conjugated Anti CD4, PE-conjugated CD8, and PE-conjugated CD25 were 
added to $10^{6}$ cells $/ \mathrm{mL}$ and incubated at $4^{\circ} \mathrm{C}$ for $30 \mathrm{~min}$ followed by washing twice with a staining buffer and resuspended in $500 \mu \mathrm{L}$ of a staining buffer. For Foxp3 staining, intracellular staining was done on the $10^{6}$ cells $/ \mathrm{mL}$ using a Biolegend True-Nuclear ${ }^{\mathrm{TM}}$ transcription factor buffer set according to the manufacturer's instructions.

\section{Statistical Analysis}

Data were analyzed using GraphPad Prism version 6 (GraphPad Software, USA). The average is shown as the mean \pm standard deviation (SD). Descriptive statistics, independent $t$-tests, and post hoc tests for one-way ANOVA were used. Also, alternative non-parametric tests of the above statistical tests were considered. Analysis of survival was done with a Mantel-Cox test. Values of $p<0.05$ were considered statistically significant.

\section{Results}

\section{Liposome Characterization}

Liposomal anti-CTLA-4 blockade antibodies with HSPC/ mPEG2000-DSPE/cholesterol (molar ratio of 55/5/40) formulations were prepared at a lipid concentration of $48 \mathrm{mM}$, according to the Bartlett phosphorus assay (Table 1). Phospholipid concentrations of liposomal formulations were in accordance with the expected lipid molar ratio. The Z-average sizes of liposomes were $166.2 \pm 5.9 \mathrm{~nm}$, with a PDI of less than 0.17. The zeta potential study showed that the HSPC/mPEG2000-DSPE formulations had the zeta potentials ( $\mathrm{mV}$ mean) of around -12 . As shown in Table 1, the mean encapsulation efficiency of the anti-CTLA-4 PEG-liposomes was around 54.7\%.

\section{Encapsulation of Anti-CTLA-4 Increased Its Therapeutic Effect}

The therapeutic effect of liposomal formulations containing the anti-CTLA-4 Antibody was assessed by the evaluation of the mice survival and tumor size. In comparison with the control group, only the liposomal anti-CTLA-4 antibody increased mice survival significantly. Tumor size regression in the liposomal anti-CTLA-4 antibody receiving mice was significant compared to both the control and non-liposomal anti-CTLA-4 antibody receiving mice. Consistent with our previous study, encapsulation of anti-CTLA-4 PEGliposomes produced a substantial benefit in the survival of mice in comparison with free the anti-CTLA-4 antibody $(P<0.0001)$ (Figure 1B-F). ${ }^{16}$ The therapeutic efficiency study also showed greater TGD and TTE in mice receiving
anti-CTLA-4 PEG-liposomes over non-liposomal antiCTLA-4 antibody (Table 2). Consistent with an increase in survival rate and reduction of tumor size in liposomal antiCTLA-4 treated mice, we observed a significant change in the immunological cell population in lymph nodes and TIL. Lymph node $\mathrm{CD}^{+}$cell populations significantly increased in the mice treated with non-liposomal and liposomal antiCTLA-4 blocking antibody compared to the control group. Despite this increase in the $\mathrm{CD}^{+}$population, $\mathrm{CD} 8^{+} /$Treg ratio remained constant (Figure $1 \mathrm{G}-\mathrm{I}$ ). In TIL, we observed a significant increase in the $\mathrm{CD}^{+}$population and $\mathrm{CD}^{+} /$Treg ratio in the study group receiving liposomal anti-CTLA-4 (Figure 1J-L).

\section{Combination Sequence of Anti-CTLA-4 with Doxil}

The therapeutic combination of the checkpoint blockers agents, such as anti-CTLA-4 antibodies, with chemotherapies and the effect of combination timing, are emerging concepts in cancer immunotherapy. To assess the effect of the sequence on the administration of anti-CTLA-4 in combination with Doxil, first, we tested a combination therapy of the non-liposomal form of anti-CTLA-4 Antibody with Doxil in different orders of administration times, e.g., before Doxil, concomitant with Doxil and after Doxil injection. We observed a significant increase in survival rate and decrease tumor size in mice receiving anti-CTLA-4 before the administration of Doxil. Concomitant treatment with anti-CTLA-4 and Doxil was only effective while mice were receiving treatment regimens. Injection of anti-CTLA-4 after Doxil showed antagonism to some degree and decreased the survival of mice (Figure 2A-F). The therapeutic efficiency study also showed the same results, with the greatest TTE and TGD $\%$ compared to the control group, in combination of antiCTLA-4 injection before Doxil administration. As we observed in the tumor size and survival analysis, only when combining the treatment with anti-CTLA-4 administration before Doxil was a significant change in the immune cell population in the tumor microenvironment observed. The treatment of tumor-bearing mice with antiCTLA-4 before Doxil led to an increase in both changes in the $\mathrm{CD}^{+}$population and $\mathrm{CD} 8^{+} /$Treg ratio. However, none of our combination treatment regimens showed any significant changes in the $\mathrm{CD}^{+}$population and $\mathrm{CD} 8^{+} /$Treg ratio in DLN and the spleen (Figure 2G-L). 
A Doxil Injection

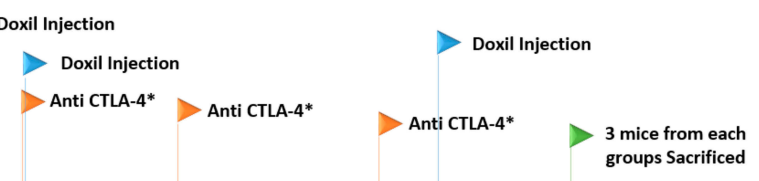

\section{\begin{tabular}{|l|l|l|l|l|l|l|l|l|l|l|l|} 
Day 0 & 3 & 5 & 7 & 9 & 11 & 13 & 15 & 17 & 19 & 21 & 23 \\
\hline
\end{tabular}}
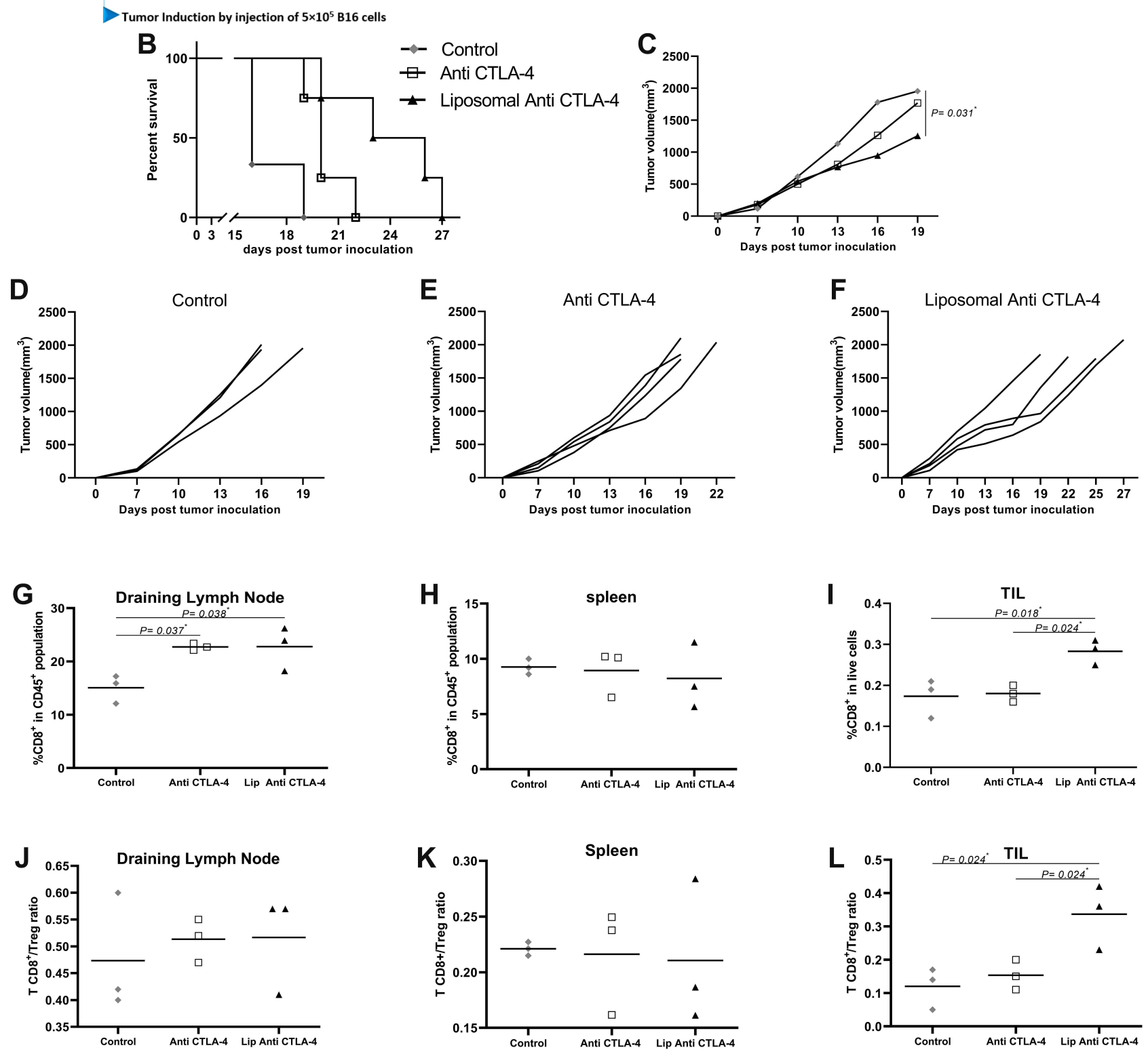

Figure I Encapsulation of liposomal enhances its antitumor effect in BI6 large established mouse model. (A) Injection schedule for all treatment groups. (B) Survival analysis mice bearing BI6 tumor, liposomal increased survival significantly, Log-rank analysis shows statistically significant differences between treatment groups. (C) Comparison between tumor size of different groups, liposomal decreased tumor size in comparison to non-liposomal and PBS. (D-F) Tumor size progression graph for each treatment groups, individually. (G) \%CD8 ${ }^{+}$in $\mathrm{CD} 45^{+}$population of DLN, increased \%CD8 ${ }^{+}$of CD $45^{+}$population in both forms. (H) \%CD8 ${ }^{+}$of in CD $45^{+}$population spleen. (I) \% $\mathrm{CD}^{+}$in live cells of the tumor microenvironment, only liposomal increased \%CD8 ${ }^{+}$in $\mathrm{TIL}$. (J) $\mathrm{TCD}^{+} / \mathrm{T}$ reg ratio in DLN. (K) $\mathrm{TCD} 8^{+} / \mathrm{T}$ reg ration in spleen. (L) $\mathrm{TCD}^{+} / \mathrm{T}$ reg ratio in TIL, only liposomal increased $\mathrm{TCD}^{+} / \mathrm{T}$ reg ration ratio $\left({ }^{*} \mathrm{P}<0.05\right)$.

Abbreviation: OXP, oxaliplatin. 
Table I Characteristics and Encapsulation Efficiencies of Encapsulated CTLA-4 Blockade Antibody Liposomal Formulations

\begin{tabular}{|c|c|c|c|c|c|c|c|}
\hline $\begin{array}{l}\text { Formulations- } \\
\text { Molar Ratio }\end{array}$ & $\begin{array}{l}\text { Total Lipid } \\
\text { Concentrations } \\
(\mathrm{mM})\end{array}$ & $\begin{array}{l}\text { Expected Total } \\
\text { Phospholipid } \\
\text { Concentrations } \\
\text { (mM) }\end{array}$ & $\begin{array}{l}\text { Observed Total } \\
\text { Phospholipid } \\
\text { Concentrations } \\
\text { (mM) }\end{array}$ & $\begin{array}{l}\text { Z Average } \\
(\mathrm{nm}) \_S D\end{array}$ & PDI ${ }^{a}$ & $\begin{array}{l}\text { Zeta } \\
\text { Potentials } \\
(\mathrm{mV}) \_ \text {SD }\end{array}$ & $\begin{array}{l}\text { Encapsulation } \\
\text { Efficacy\% }\end{array}$ \\
\hline $\begin{array}{l}\text { PEGylated } \\
\text { liposome: HSPC/ } \\
\text { mPEG2000- DSPE/ } \\
\text { Chol 55/5/40 }\end{array}$ & 48 & 28.8 & $24.6 \pm 1.7$ & $166.2 \pm 5.9$ & $\begin{array}{l}0.16 \\
\pm 0.063\end{array}$ & $9.14 \pm 2.6$ & $54.7 \pm 8.9$ \\
\hline
\end{tabular}

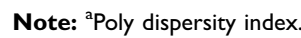

\section{Combination of Liposomal Anti-CTLA-4 with Doxil}

To assess whether liposomal anti-CTLA-4 synergized with Doxil in the combining therapy, and considering the result from the combination therapy with non-liposomal antiCTLA-4, we injected liposomal anti-CTLA-4 before Doxil treatment. As shown in Figure 3A-E, combining liposomal anti-CTLA-4 plus Doxil increased survival rates and decreased tumor size in comparison with the non-liposomal anti-CTLA-4 plus Doxil receiving group. Consistent with the survival study, the therapeutic efficiency study showed the same results. Combining the therapy using liposomal anti-CTLA-4 led to an increase in $\mathrm{CD}^{+} /$Treg ratio in DLN and tumor microenvironment, in spite of failing to see a significant increase in the $\mathrm{CD} 8^{+}$ population (Figure 3F-K).

\section{Discussion}

Here, we developed an anti-CTLA-4 antibody containing PEGylated liposome. In our previous study, we showed that it accumulates in tumors remarkedly more than the non-liposomal blocking CTLA-4 antibody and has

Table 2 Therapeutic Efficacy Data of Therapeutic and Control Group Mice Bearing BI6 Tumor

\begin{tabular}{|c|c|c|}
\hline Groups & $\begin{array}{l}\text { TTE }^{\mathrm{a}} \text { (Days) } \\
\text { 土SD }\end{array}$ & $\begin{array}{l}\text { TGD }^{\mathbf{b}} \\
(\%)\end{array}$ \\
\hline Control & $16.01 \pm 1.14$ & - \\
\hline Anti-CTLA-4 & $19.67 \pm 2.38$ & 22.86 \\
\hline Liposomal Anti-CTLA-4 & $33.34 \pm 2.63$ & 113.3 \\
\hline Doxil & $22.2 \pm 2.18$ & 39.04 \\
\hline Anti-CTLA-4 + Doxil & $41.84 \pm 1.93$ & 161.32 \\
\hline $\begin{array}{l}\text { Concomitant Anti-CTLA-4 and } \\
\text { Doxil }\end{array}$ & $32.43 \pm 2.13$ & 102.56 \\
\hline Doxil+ Anti-CTLA-4 & $23.78 \pm 3.63$ & 48.51 \\
\hline Doxil +liposomal Anti-CTLA-4 & $42.86 \pm 4.45$ & 167.69 \\
\hline
\end{tabular}

Notes: ${ }^{\mathrm{a}}$ Time to reach end point. ${ }^{\mathrm{b}}$ Tumor growth delay. a prolonged blood half-life. Moreover, its therapeutic effect was studied using a syngenic C-26 tumor mouse model. Liposomal anti-CTLA-4 led to an increase in survival and reduction in tumor size of tumor-bearing mice and increased $\mathrm{CD}^{+}$and $\mathrm{CD} 8^{+} /$Treg ratio in the tumor microenvironment. ${ }^{16}$

In this study, we evaluated the therapeutic efficiency of liposomal anti-CTLA-4 in large established tumors from a B16 mouse model of melanoma. As with our previous research, the encapsulation of an anti-CTLA- 4 antibody led to an increase in survival and a reduction in tumor size. There is little evidence for the use of nanoparticles in delivering checkpoint blockers. For instance, Ordikhani et al used PLGA nanoparticles as carriers for anti-PD-1 delivery. They found an increase in tumor accumulation of encapsulated anti-PD-1 in the tumor, which translated into an increase in survival and reduction of tumor size. In spite of this increase in tumoral accumulation and better response, it led to an increase in mortality of mice receiving these nanoparticles as a result of the hyperactivation of T cells. ${ }^{17} \mathrm{We}$ did not find this kind of side effect in any of our treatment groups. This may be due to different mechanisms of anti-PD-1 and anti-CTLA-4.

The anti-CTLA-4 antibody is an immune checkpoint blocker which has demonstrated impressive results in the treatment of several types of cancers. ${ }^{18}$ However, the toxicity of immune checkpoint blockers known as irAEs, especially, has been shown to be a significant problem in managing patients receiving these blockers. These irAEs include common manifestations such as dermatological, gastrointestinal, and endocrine effects on the involvement of nervous, hematopoietic, and urinary systems, which are rarer disorders. ${ }^{19}$ Besides their frequency, these adverse events can be lethal. Recently by utilizing engineered mAbs, the rate of irAEs has been reduced significantly. However, several limitations, such as poor tissue penetration as a result of the large size of the antibodies, might 

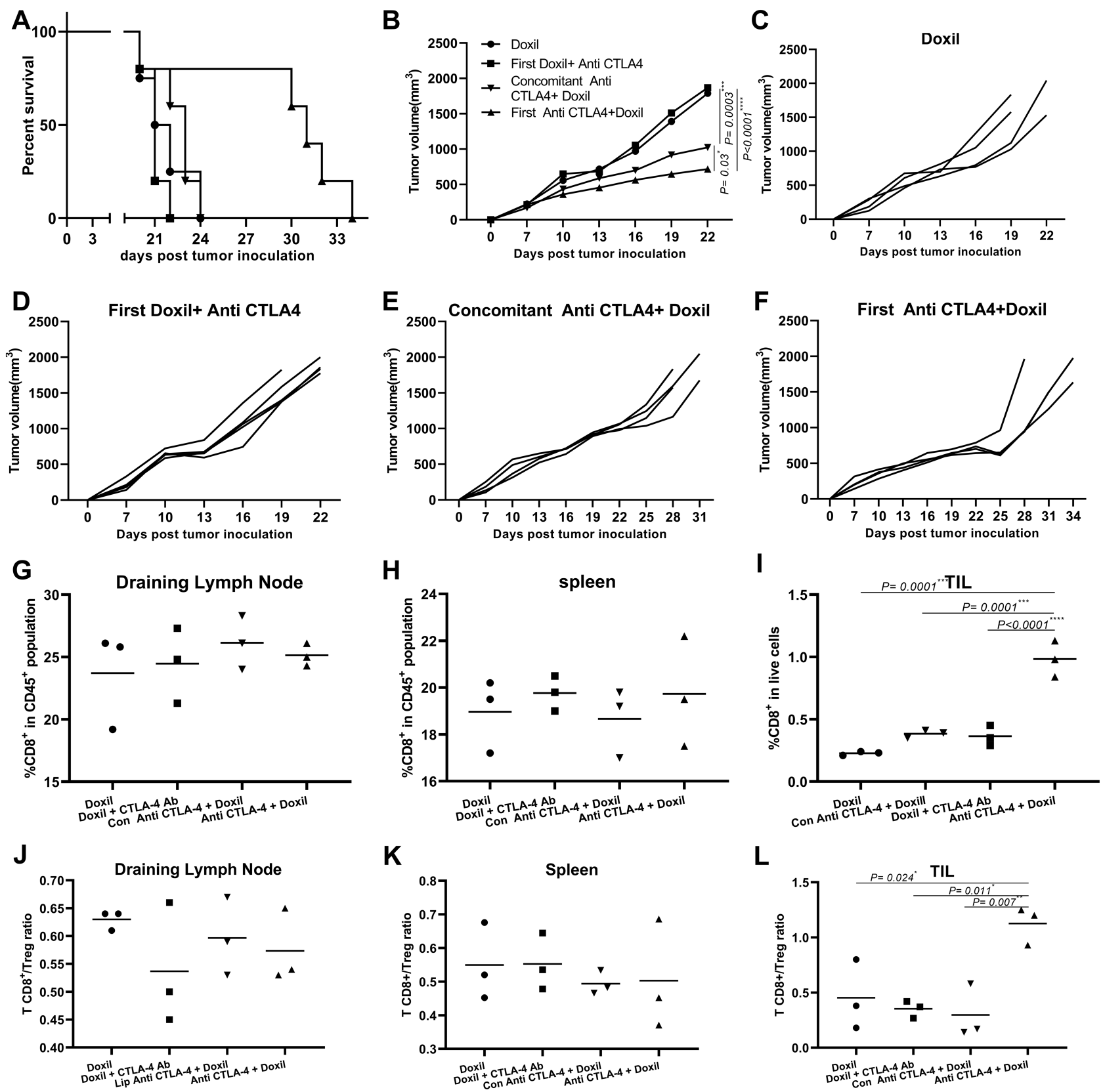

Figure 2 Administration before Doxil in combination therapy shows promising results in BI6 large established mouse model. (A) Survival analysis - mice bearing BI6 tumor, injection prior to Doxil increased survival significantly, Log-rank analysis shows statistically significant differences between treatment groups. (B) Comparison between tumor size of different groups, combining therapy in sequence of before Doxil reduced tumor size in comparison to other groups. (C-F) Tumor size progression graph for each treatment groups, individually. (G) $\% \mathrm{CD}^{+}$in $\mathrm{CD} 45+$ population of DLN. (H) $\% \mathrm{CD} 8^{+}$of in $\mathrm{CD} 45^{+}$population spleen. (I) \%CD8 ${ }^{+}$in live cells of tumor microenvironment, only of anti-CTLA-4 + Doxil (in sequence manner) increased \%CD8 ${ }^{+}$in TIL. (J) $\mathrm{T} \mathrm{CD8}^{+} / \mathrm{T}$ reg ratio in DLN. (K) TCD8 ${ }^{+} / \mathrm{T}$ reg ration in spleen. (L) $\mathrm{TCD}^{+} / \mathrm{T}$ reg ratio in TIL, only administration before Doxil increased TCD8 ${ }^{+} / \mathrm{T}$ reg ration ratio $\left({ }^{*} \mathrm{p}<0.05,{ }^{* *} \mathrm{p}<0.0 \mathrm{I}, *^{* * *} \mathrm{p}<0.00 \mathrm{I}, * * * * \mathrm{p}<0.000 \mathrm{I}\right)$.

have impeded their usage as common therapeutic agents. To overcome this disadvantage, mAb injection often takes place several times, which raises the cost and discomfort of patients. Using nanoparticles will overcome these challenges and also enable site-specific accumulation of checkpoint blockers. ${ }^{20}$ PEGylated liposomes have been approved by the FDA and have strong potential for clinical usage.
Anti-CTLA-4 effects and limits early immune system response and mainly leads to a reduction in the number and alteration of the function of Tregs. While anti-PD-1 acts in the effector immune response phase and interferes with more T-cell activation signals, it has a widespread effect. Another group encapsulated anti-PD-1 in a photosensitive liposomal nanocarrier in the B16 melanoma mouse model, which led to a better tumor response and an increase in the infiltration of 
A

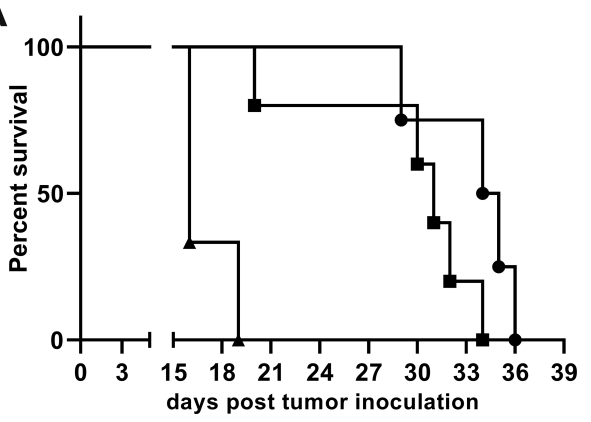

C

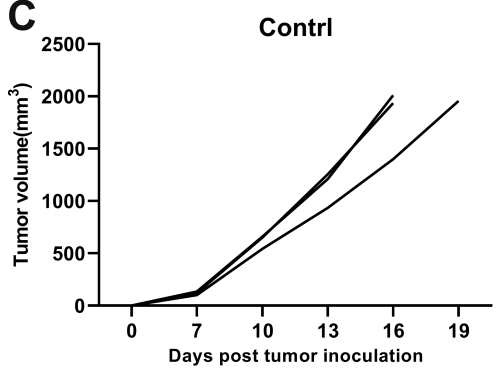

F

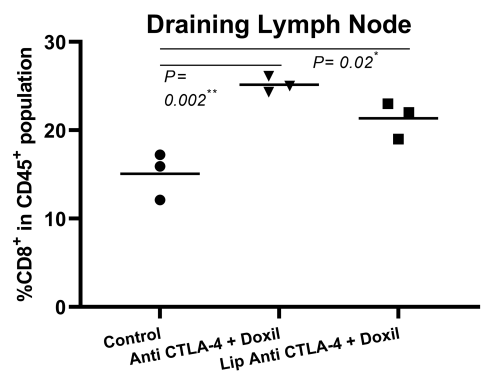

I

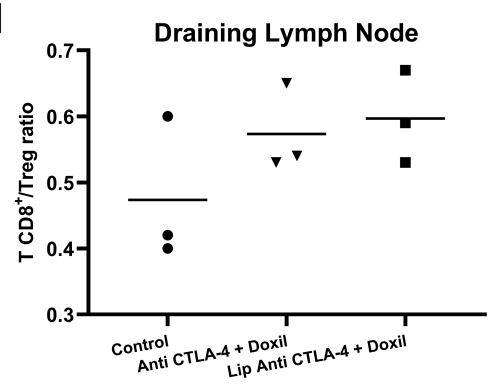

\section{D}

G

J
B
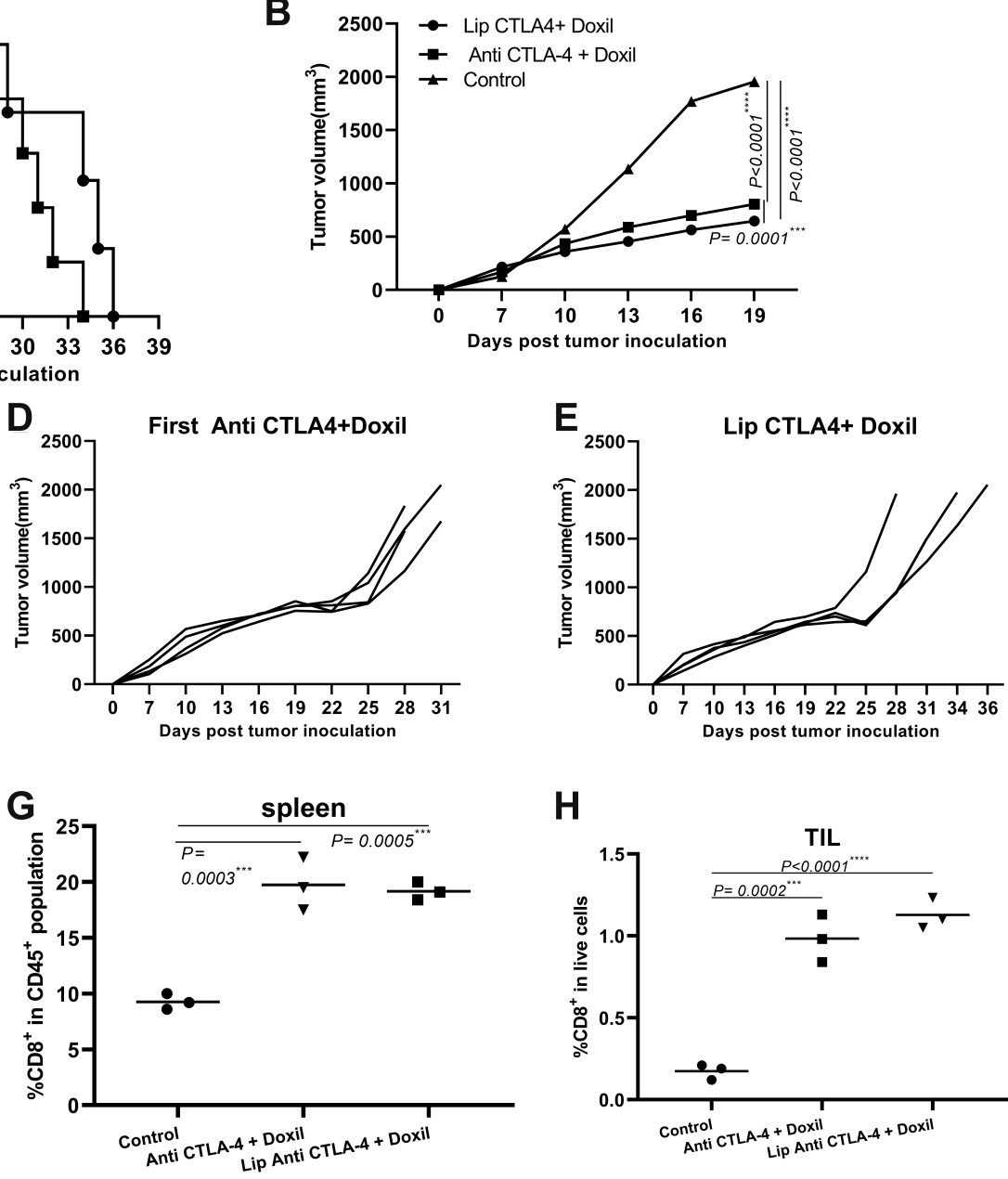

H
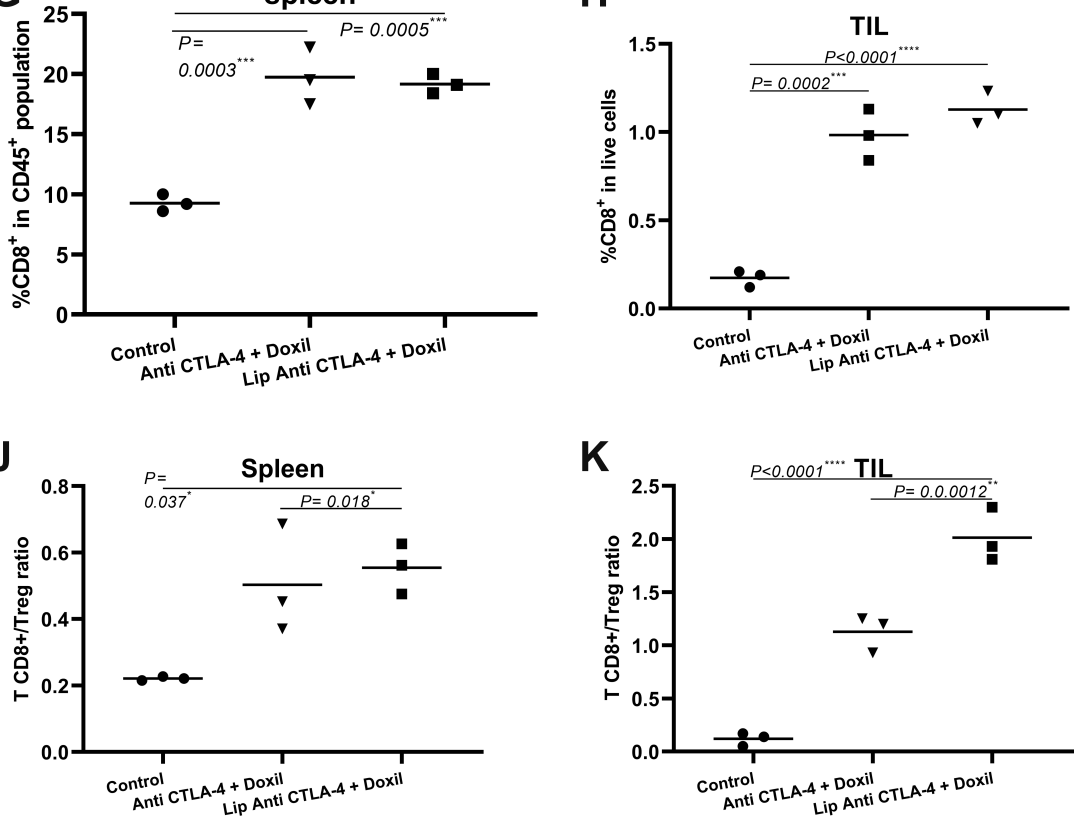

K

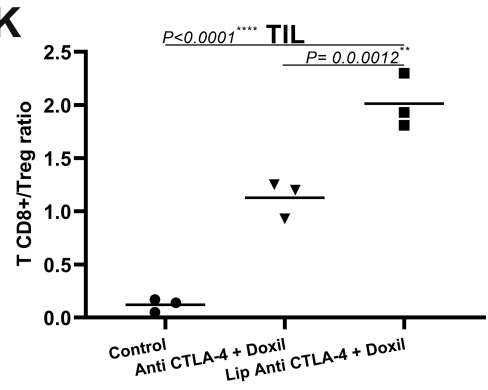

Figure 3 Liposomal delivery increases the efficiency of combination therapy of anti-CTLA-4 with Doxil. (A) survival analysis mice bearing BI6 tumor, liposomal increases survival significantly in combination therapy when replaced with non-liposomal treatment, Mantel-Cox test shows statistically significant differences between treatment groups $(p<0.001)$. (B) Comparison between tumor size of different groups, combining therapy with liposomal before Doxil reduced the tumor size in comparison to other groups. (C-E) Tumor size progression graph for each treatment groups, individually. (F) \%CD8 ${ }^{+}$in $C D 45^{+}$population of DLN. (G) \%CD8 ${ }^{+}$of in $C D 45^{+}$population spleen.

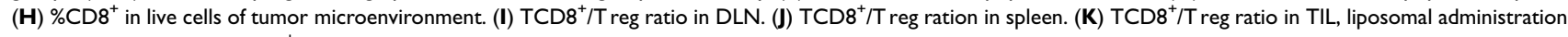
before Doxil increased TCD8 ${ }^{+} / \mathrm{T}$ reg ration ratio compared to non-liposomal treatment $\left({ }^{*} \mathrm{p}<0.05,{ }^{* *} \mathrm{p}<0.0 \mathrm{I}, * * * \mathrm{p}<0.00 \mathrm{I}, * * * * \mathrm{p}<0.000 \mathrm{I}\right)$.

$\mathrm{CD}^{+} \mathrm{T}$ cells. As they utilized controlled release by thermal mechanisms, they did not report any significant side effects. $^{21}$

Today's combinational approach in cancer therapy is used more frequently, as almost all of the current protocols in cancer treatment have failed to eliminate tumor cells when using monotherapies. We previously reported a beneficial combination of anti-PD-L1 with oxaliplatin in CT-26 colorectal mouse. ${ }^{22}$ Rios-Diora et al showed that the combination of anti-CTLA-4 and Anti PD-L1 with Doxil and doxorubicin increases anti-tumor efficiency in both small and established CT-26 tumor models. ${ }^{5}$ There are 
several studies using a combination of anti-CTLA-4 and chemotherapy in tumor models other than CT-26. Wu et al showed that the combination of anti-CTLA-4 with Cisplatin produced a better therapeutic response than monotherapy alone in Murine Mesothelioma. Furthermore, it expands infiltrating $\mathrm{T}$ Cells in the tumor microenvironment. ${ }^{23}$ Another group used the combination of anti-CTLA-4 with Gemcitabine in a EMT-6/P breast cancer model. They concluded that metronomic chemotherapy has a better response in combination with anti-CTLA-4. ${ }^{24}$ Winograd et al used anti-CTLA-4 in combination with encapsulated paclitaxel and Gemcitabine and showed that combination therapy increases the efficiency of the therapy remarkably. This effect was due to the $\mathrm{CD} 8^{+} \mathrm{T}$ cell population. ${ }^{25}$

Our results showed that the combination of anti-CTLA -4 with Doxil depends on the sequence of administration. Injection of anti-CTLA-4 after Doxil showed antagonism and the survival of mice reduced. However, for tumor size, the difference was not significant until the injection of the Antibody was stopped based on the scheduled injection timetable. When anti-CTLA-4 with Doxil injection was concomitant, there was no difference in survival between monotherapy with Doxil and a combination therapy. The only sequence of injection which showed synergy when combining anti-CTLA-4 with Doxil was the administration of Doxil after anti-CTLA-4.

The therapeutic combination in cancer treatment depends on the sequence of combined-therapeutic strategies. ${ }^{26}$ Our data suggest that the synergy between anti-CTLA-4 and Doxil occurs only when we inject antiCTLA-4 prior to Doxil. Our previous experiment on assessing the efficiency of combining therapy using oxaliplatin and anti-PD-L1 also showed the importance of checkpoint blocker administration when combining it with chemotherapy, with a better response when anti-PD-L1 was injected before oxaliplatin. ${ }^{22}$ There is only one study that assessed the effect of timing in combining therapies using antiCTLA-4 with GM-CSF-secreting vaccine (GVAX). In contrast to our results, they showed a beneficial effect of combination therapy when anti-CTLA-4 was injected after GVAX $^{27}$ We used a chemotherapeutic agent which has a different mechanism of action from GVAX. As our results demonstrated when we used anti-CTLA-4 before Doxil in a combination treatment, there was a marked increase in $\mathrm{TCD} 8^{+} /$Treg ratio in the TIL population, which resembled the mechanism of action of anti-CTLA -4. Recent evidence showed that blockade of the CTLA-4 with an antibody will result in depletion of tumor- infiltrating Tregs. ${ }^{28}$ The depletion of Tregs only occurred if we administered anti-CTLA-4 prior to the Doxil treatment in the combination regimen and was enhanced when the combination was based on encapsulated anti-CTLA-4 plus Doxil. These findings are very important clinically as many patients will receive chemotherapy prior to the administration of immunotherapy. Our study suggests that further consideration should be given to the order of treatment in patients to maximize overall success and obtain synergy.

\section{Conclusions}

In the current study, we showed that encapsulating the anti-CTLA-4 antibody in PEGylated liposomes leads to a better response either in monotherapy or combination therapy in a B16 mouse model of melanoma. Furthermore, administration of the anti-CTLA-4 before Doxil in combination therapy showed a better overall response and a more favorable immune cell shift in the tumor microenvironment by a significant increase in the $\mathrm{T} \mathrm{CD} 8^{+} / \mathrm{T}$ reg ratio in our tumor model. Our data support the idea of encapsulating anti-CTLA-4 and other checkpoint blockers in nanoparticles to increase their potential in tumor accumulation and treatment of cancer. However, further studies are essential for assessing possible side effects. Furthermore, once again, we clarify the beneficial effect of the combination therapy of checkpoint blockers with conventional chemotherapies.

\section{Acknowledgment}

This work was funded by Mashhad University of Medical Sciences (930574) and Shahid Beheshti University of Medical Sciences (16935).

\section{Disclosure}

The authors declare no conflicts of interests in this work.

\section{References}

1. Gebremeskel S, Johnston B. Concepts and mechanisms underlying chemotherapy induced immunogenic cell death: impact on clinical studies and considerations for combined therapies. Oncotarget. 2015;5. doi:10.18632/oncotarget.6113

2. Yamazaki T, Hannani D, Poirier-Colame V, et al. Defective immunogenic cell death of HMGB1-deficient tumors: compensatory therapy with TLR4 agonists. Cell Death Differ. 2014;21(1):69. doi:10.1038/ cdd. 2013.72

3. Galluzzi L, Buqué A, Kepp O, et al. Immunogenic cell death in cancer and infectious disease. Nat Rev Immunol. 2017:17(2)2-5.

4. Zitvogel L, Apetoh L, Ghiringhelli F, et al. Immunological aspects of cancer chemotherapy. Nat Rev Immunol. 2008;8(1):59-73. doi:10. $1038 /$ nri2216 
5. Rios-Doria J, Durham N, Wetzel L, et al. Doxil synergizes with cancer immunotherapies to enhance antitumor responses in syngeneic mouse models. Neoplasia. 2015;17(8):661-670. doi:10.1016/j. neo.2015.08.004

6. Green AE, Rose PG. Pegylated liposomal doxorubicin in ovarian cancer. Int J Nanomedicine. 2010;363(3):711-723.

7. Van Der Kraak L, Goel G, Ramanan K, et al. 5-Fluorouracil upregulates cell surface B7-H1 (PD-L1) expression in gastrointestinal cancers. J Immunother Cancer. 2016;4(1):65. doi:10.1186/s40425016-0163-8

8. Pfirschke C, Engblom C, Rickelt S, et al. Immunogenic chemotherapy sensitizes tumors to checkpoint blockade therapy. Immunity. 2016;44(2):343-354. doi:10.1016/j.immuni.2015.11.024

9. Pardoll DM. The blockade of immune checkpoints in cancer immunotherapy. Nat Rev Cancer. 2012;12(4):252-264. doi:10.1038/ nrc3239

10. Hodi FS, O'Day SJ, McDermott DF, et al. Improved survival with ipilimumab in patients with metastatic melanoma. $N$ Engl J Med. 2010;363(8):711-723. doi:10.1056/NEJMoa1003466

11. Robert C, Thomas L, Bondarenko I, et al. Ipilimumab plus dacarbazine for previously untreated metastatic melanoma. $N$ Engl J Med. 2011;364(26):2517-2526. doi:10.1056/NEJMoa1104621

12. Bertrand A, Kostine M, Barnetche T, et al. Immune related adverse events associated with anti-CTLA-4 antibodies: systematic review and meta-analysis. BMC Med. 2015;13(1):211. doi:10.1186/s12916015-0455-8

13. Weber J. Review: anti-CTLA-4 antibody ipilimumab: case studies of clinical response and immune-related adverse events. Oncologist. 2007;12(7):864-872. doi:10.1634/theoncologist.12-7-864

14. Hansel TT, Kropshofer H, Singer T, et al. The safety and side effects of monoclonal antibodies. Nat Rev Drug Discov. 2010;9(4):325. doi: $10.1038 / \mathrm{nrd} 3003$

15. Nikpoor AR, Tavakkol-Afshari J, Gholizadeh Z, et al. Nanoliposomemediated targeting of antibodies to tumors: IVIG antibodies as a model. Int J Pharm. 2015;495(1):162-170. doi:10.1016/j.ijpharm. 2015.08.048

16. Nikpoor AR, Tavakkol-Afshari J, Sadri K, et al. Improved tumor accumulation and therapeutic efficacy of CTLA-4-blocking antibody using liposome-encapsulated antibody: in vitro and in vivo studies. Nanomedicine. 2017;13(8):2671-2682. doi:10.1016/j.nano.2017.08. 010

17. Ordikhani F, Uehara M, Kasinath V, et al. Targeting antigenpresenting cells by anti-PD-1 nanoparticles augments antitumor immunity. JCI Insight. 2018;3(20). doi:10.1172/jci.insight.122700
18. Seidel JA, Otsuka A, Kabashima K. Anti-PD-1 and anti-CTLA-4 therapies in cancer: mechanisms of action, efficacy, and limitations. Front Oncol. 2018;8:1-14. doi:10.3389/fonc.2018.00086

19. Kumar V, Chaudhary N, Garg M, et al. Current diagnosis and management of Immune Related Adverse Events (irAEs) induced by immune checkpoint inhibitor therapy. Front Pharmacol. 2017;8:49. doi:10.3389/fphar.2017.00049

20. Patnaik A, Kang SP, Rasco D, et al. Phase I study of pembrolizumab (MK-3475; Anti-PD-1 monoclonal antibody) in patients with advanced solid tumors. Clin Cancer Res. 2015;21(19):4286-4293. doi:10.1158/1078-0432.CCR-14-2607

21. Zhang N, Song J, Liu Y, et al. Photothermal therapy mediated by phase-transformation nanoparticles facilitates delivery of anti-PD1 antibody and synergizes with antitumor immunotherapy for melanoma. J Control Release. 2019;306:15-28. doi:10.1016/j.jconrel.2019.05.036

22. Golchin S, Alimohammadi R, Rostami Nejad M, et al. Synergistic antitumor effect of anti-PD-L1 combined with oxaliplatin on a mouse tumor model. J Cell Physiol. 2019;234(11):19866-19874. doi:10.1002/ jcp. 28585

23. Wu L, Yun Z, Tagawa T, et al. CTLA-4 blockade expands infiltrating $\mathrm{T}$ cells and inhibits cancer cell repopulation during the intervals of chemotherapy in murine mesothelioma. Mol Cancer Ther. 2012;11 (8):1809-1819. doi:10.1158/1535-7163.MCT-11-1014

24. Parra K, Valenzuela P, Lerma N, et al. Impact of CTLA-4 blockade in conjunction with metronomic chemotherapy on preclinical breast cancer growth. Br J Cancer. 2017;116(3):324-334. doi:10.1038/bjc.2016.429

25. Winograd R, Byrne KT, Evans RA, et al. Induction of T-cell immunity overcomes complete resistance to PD-1 and CTLA-4 blockade and improves survival in pancreatic carcinoma. Cancer Immunol Res. 2015;3(4):399-411. doi:10.1158/2326-6066.CIR-14-0215

26. Nejad EB, Welters MJP, Arens R, et al. Expert opinion on biological therapy the importance of correctly timing cancer immunotherapy. Expert Opin Biol Ther. 2017;17(1):87-103. doi:10.1080/ 14712598.2017.1256388

27. Sutmuller RPM, Van Duivenvoorde LM, Van Elsas A, et al. Synergism of cytotoxic T lymphocyte-associated antigen 4 blockade and depletion of $\mathrm{Cd} 25+$ regulatory $\mathrm{T}$ cells in antitumor therapy reveals alternative pathways for suppression of autoreactive cytotoxic T lymphocyte responses. J Exp Med. 2001;194(6):823-832. doi:10.1084/jem.194.6.823

28. Simpson TR, Li F, Montalvo-Ortiz W, et al. Fc-dependent depletion of tumor-infiltrating regulatory $\mathrm{T}$ cells co-defines the efficacy of antiCTLA-4 therapy against melanoma. $J$ Exp Med. 2013;210 (9):1695-1710. doi:10.1084/jem.20130579
International Journal of Nanomedicine

\section{Publish your work in this journal}

The International Journal of Nanomedicine is an international, peerreviewed journal focusing on the application of nanotechnology in diagnostics, therapeutics, and drug delivery systems throughout the biomedical field. This journal is indexed on PubMed Central, MedLine, CAS, SciSearch ${ }^{\mathbb{B}}$, Current Contents ${ }^{\mathbb{B}} /$ Clinical Medicine, $^{2}$
Journal Citation Reports/Science Edition, EMBase, Scopus and the Elsevier Bibliographic databases. The manuscript management system is completely online and includes a very quick and fair peer-review system, which is all easy to use. Visit http://www.dovepress.com/ testimonials.php to read real quotes from published authors. 